\title{
Maternal autoimmune disease and the fetus
}

The privileged position of the fetus, containing foreign protein that is not rejected by its mother, has intrigued biologists for many years. More recently, it has been realised that certain maternal autoimmune diseases, particularly immune thrombocytopenia, Graves' disease, pemphigoid gestationis (herpes gestationis), myasthenia gravis, and systemic lupus erythematosus may affect the fetus by immune mechanisms, and, in particular, that the degree of fetal involvement may be out of proportion to the extent of maternal illness. Rhesus isoimmunisation has been specifically excluded from the following discussion sinee, although the fetus is affected by maternal antibody, this antibody is not related to maternal autoimmune disaese.

\section{Immune thrombocytopenia}

In immune thrombocytopenia, IgG antibodies may cross the placenta and cause thrombocytopenia and subsequent risk of bleeding in the fetus. The overall risk of subsequent neonatal thrombocytopenia is about $45 \%$. When in pregnancy transplacental passage of antibodies occurs is not known. Although the risk of bleeding is greatest during labour, where catastrophic intracerebral haemorrhage can be fatal to the fetus, the fetus may have thrombocytopenia well before this stage. Most believe that those fetuses at risk should be delivered by caesarean section, although this is disputed by others.

The quantification of fetal risk has been based on the measurement of free antiplatelet $\operatorname{IgG}$ in the serum as detected by the absorption of IgG from maternal plasma to normal platelets (indirect test). ${ }^{1}$ This has been said to correlate well with neonatal thrombocytopenia, and could represent a considerable advance over previous tests based on measurement of free IgG in maternal plasma or the absolute platelet count. The absolute platelet count is particularly unreliable, since patients in clinical remission may still have high antibody titres and therefore be at risk of producing an affected fetus.

Standard treatment for immune thrombocytopenia has been with steroids, splenectomy (best performed before pregnancy), and immunosupressive drugs. With the possible exception of azathioprine, immunosupressive drugs should not be used in pregnancy. The use of large doses of intravenous human IgG, however, represents a real advance in short term treatment. ${ }^{2}$ This has improved maternal platelet count in pregnancy, and will probably improve fetal outcome.

\section{Graves' disease}

There is good evidence that the pathophysiology of Graves' disease is intimately related to the presence of thyroid stimulating autoantibodies that bind to thyroid stimulating hormone receptors in the thyroid cell and cause excessive secretion of thyroid hormones. These autoantibodies also cross the placenta and can stimulate the fetal thyroid gland, causing intrauterine and neonatal hyperthyroidism in up to $10 \%$ of women with a past or current history of Graves' disease. Dirmikis and Munro $^{3}$ have shown that the presence of more than $20 \mathrm{U} / \mathrm{ml}$ long acting thyroid stimulator-protector accurately predicts the subsequent development of neonatal thyrotoxicosis. The titre of thyroid stimulating autoantibodies does not necessarily correlate with the thyroid status of the mother. Thus it is possible for a hyperthyroid baby to be born to a woman who is euthyroid, or hypothyroid, but has had Graves' disease, and who has a high antibody titre. Nevertheless, there are problems with this relatively simplistic approach: in the neonate, the half life of antibodies that have crossed the placenta is believed to be 5 to 14 days, ${ }^{4}$ yet neonatal hyperthyroidism may persist for several years. One child who is an identical twin may have neonatal hyperthyroidism; the other may not. Three infants with neonatal Graves' disease have been described where there was no history of thyroid disease in the mother. Delayed onset ( 2 to 4 months) Graves' disease has been reported in two infants of a mother with Hashimoto's disease whose serum did not have long acting thyroid stimulator or its protector, but did have a thyroid stimulating autoantibody inhibitor. ${ }^{4}$ Thus, it seems that both the genotype and phenotype of the child are important in the expression of Graves' disease in the neonate, and also that the occurence of Graves' disease in the neonate (and it's mother) may depend on a balance between thyroid stimulating autoantibodies and an inhibitor. In addition, some cases of congenital hypothyroidism seem to be related to a maternal immunoglobulin which blocks thyroid stimulating hormone in the absence of thyroid dysfunction in the mother. ${ }^{5}$

The recognition of Graves' disease in utero is complicated because both immunoglobulins and 
antithyroid drugs given to the mother to treat the condition may affect the fetus in opposing directions. A persistent fetal tachycardia greater than 150 beats per minute, sometimes associated with ultrasound evidence of heart failure, and intrauterine growth retardation suggest a fetus with hyperthyroidism. These fetuses have been treated in utero by carbimazole given to the mother with additional thyroxine, to maintain her thyroid status.

\section{Pemphigoid gestationis (herpes gestationis)}

This is a very uncommon skin condition present in about 1 in 50000 pregnancies, ${ }^{6}$ but almost specific to pregnancy. It is characterised by subepidermal blisters that often start around the umbilicus. It is almost certainly caused by an autoantibody, since skin biopsy shows IgG and C3 complement deposition in the basal cell plasma membrane ${ }^{6}$ and since the serum of these patients contains IgG immunoglobulin that binds to the basement membrane zone of the skin. The intense itching and severe constitutional effects may be successfully treated with oral steroids, initially in high dosage, or by plasma exchange. The fetus may be affected by a similar transient eruption, indicating transplacental passage of the antibodies. A recent study showed no increased perinatal mortality, but a high incidence of growth retardation. ${ }^{7}$

\section{Myasthenia gravis}

This condition is caused by IgG antibodies that bind to receptors on the motor end plate, blocking neuromuscular transmission and causing weakness of voluntary but not smooth muscle. The IgG antibodies cross the placenta and the neonate is temporarily affected in up to $20 \%$ of cases. ${ }^{8}$ Indeed, the existence of neonatal myasthenia was one of the major clues to unravelling the pathophysiology of this condition.

The mainstay of treatment is anticholinesterase drugs such as neostigmine and pyridostigmine. These drugs should be given parenterally in labour. Corticosteroids, plasmapheresis (for crises), thymectomy, and antimetabolites are also used, but the latter two are not recommended in pregnancy.

The fetus is not affected in utero; perhaps transplacental passage of maternal drugs or the action of certain components of amniotic fluid, in particular $\alpha$ fetoprotein, in absorbing antiacetylcholine receptor antibodies accounts for this effect. The fetus is likely, however, to be born prematurely or growth retarded, and about $40 \%$ of all myasthenic pregnancies have one or both of these complications. ${ }^{9}$ Although the perinatal mortality has been reported to be as high as 82 per 1000 in retrospective surveys, ${ }^{9}$ more recent reports give a more favourable prognosis.

Early stages of labour proceed normally in patients with myasthenia because the uterus has smooth not striated muscle. The later stages, however, where maternal effort is important may be delayed.

Antiacetycholine receptor antibodies may be secreted in breast milk, and therefore the mother with very high titre antibodies or who is taking large quantities of anticholinesterase drugs should breast feed with caution. It has been stated, however, ${ }^{10}$ that anticholinesterase drugs do not enter breast milk.

Neonatal myasthenia gravis responds to anticholinesterase drugs; it only lasts about three weeks, the presumed half life of maternal antibody in the neonate. Exchange transfusion has also been used, and plasmapheresis may be helpful. ${ }^{8}$

\section{Systemic lupus erythematosus}

This is a multisystem disease which most frequently presents in young women. It is therefore relatively common in pregnancy, and it is certainly the connective tissue disease that has been studied most intensively. The apparent prevalence has increased as more mild forms of the disease are recognised.

There are three main ways in which systemic lupus erythematosus affects pregnancy and it's outcome. It increases the risk of abortion, increases the risk of late pregnancy losses due to hypertension and renal failure, and is an important cause of heart block and other cardiac defects in the newborn. This latter effect may be part of a more general neonatal lupus syndrome.

The incidence of abortion in patients with systemic lupus erythematosus may be as high as $40 \%{ }^{11}$ On reviewing previous pregnancies, even tefore the clinical onset of the disease, Fraga found that the incidence of abortion was $23 \%$, about twice as high as in a group of control patients. ${ }^{11}$ The risk of abortion is clearly not related to the severity of the condition. In systemic lupus erythematosus, abortion often occurs later than the usual 12 to 14 weeks' gestation, and, indeed, may occur at any gestation up to 28 weeks. Systemic lupus erythematosus should always be considered as an important, though rare, cause of recurrent late abortion: many of these cases have serological evidence, but no other clinical manifestations. A high titre of lymphocytotoxic antibodies has been found in patients with this disorder, whose pregnancies ended in abortion. These antibodies can be absorbed by trophoblast, which suggests a number of possible 
mechanisms for the cause of abortion. For example, necrotising decidual vascular lesions occur with immunoglobulin deposition in placentas of women whose pregnancies were complicated by systemic lupus erythematosus.

An alternative approach has been to examine the relation between the lupus inhibitor and recurrent abortion. Five to $10 \%$ of these patients develop an inhibitor of the coagulation pathway that causes prolongation of the partial thromboplastin or prothrombin times. The presence of this antibody seems to be a major risk factor for fetal loss (and paradoxically for maternal thromboembolism). Since lupus anticoagulant may be present without any other evidence of the disease, it should be looked for in all cases of recurrent abortion. The titre of anticardiolipin antibodies is highly correlated with lupus anticoagulant activity. ${ }^{12}$ In future, anticardiolipin titre may be a better index for prognosis and treatment.

There is evidence that treatment of the lupus inhibitor may improve pregnancy outcome, Lubbé ${ }^{13}$ studied a group of 10 women with 25 previous intrauterine deaths. Treatment with prednisone (40 to $60 \mathrm{mg} /$ day), and aspirin ( $75 \mathrm{mg} /$ day) resulted in five live infants from six pregnancies. It is clear that if steroids do improve the pregnancy outcome, high doses may be necessary, and this may account for previous failures of steroid treatment. ${ }^{11}$ This is, however, a form of treatment where we urgently need controlled clinical trials. Other treatments that have been used include plasmapharesis and azathioprine. In addition, to determine the precise importance of lupus anticoagulant, more information is needed on its presence in patients who do not have systemic lupus erythematosus or a bad obstetric history.

The neonatal lupus syndrome includes haematological complications, cardiac abnormalities, babies in whom discoid skin lesions are the only abnormalities, and neonates who develop the disorder whose mothers are not affected. ${ }^{14}$ In systemic lupus erythematosus, however, the precise antibody that affects the fetus has not been identified, and the fetal outcome cannot be correlated with fetal (or maternal) antibody titres. The haematological abnormalities are haemolytic anaemia, leucopoenia, and thrombocytopenia. They are usually transient, and not a major problem. By far the most common cardiac abnormality is complete heart block which may be present and detected antenatally. Although most infants born to affected mothers are normal, about one in three mothers, who deliver babies with congenital heart block have, or will have, a connective tissue disease. Most frequently the disease is systemic lupus erythematosus, but in $16 \%$ of cases the mother has had rheumatoid arthritis, and, in $25 \%$, a less well defined form of connective tissue disease. Congenital heart block has also been reported with Sjögren's syndrome.

About $60 \%$ of mothers who deliver a child with congenital heart block have anti- $\mathrm{R}_{\mathrm{o}}$ antibodies. In one series, these autoantibodies were invariably present in the mothers with systemic lupus erythematosus that delivered an affected child, but they were also present in some of those asymptomatic women who had a child with congenital heart block. ${ }^{15}$ There is therefore strong circumstantial evidence to implicate the anti- $R_{o}$ system in the pathogenesis of congenital heart block. Immunoglobulin has recently been found in the atria of a fatal case of congenital heart block. ${ }^{16}$ The neonate usually survives the perinatal period, however, and often does not require pacing. Fatal cases may also be associated with endomyocardial fibrosis or pericarditis.

The study of immune mechanisms in pregnancy (with the notable exception of haemolytic disease of the newborn) has not offered much to the clinician until recently. The maternal diseases described above are of real importance, however, both to obstetricians and paediatricians. Furthermore, the finding that immunotherapy may help a group of patients with recurrent abortion, who share many histocompatibility antigens with their husbands, ${ }^{17}$ emphasises the importance that immunology will have in reproductive medicine in the future.

\section{References}

1 Cines DB, Dusak B, Tomaski A, Mennuti M, Schreiber AD. Immune thrombocytopenic purpura and pregnancy. $N$ Engl $J$ Med 1982;306:826-31.

${ }^{2}$ Newland AC, Boots MA, Patterson KG. Intravenous IgG for auto-immune thrombocytopenia in pregnancy. $N$ Engl $J$ Med 1984;310:261-2.

${ }^{3}$ Dirmikis SM, Munro DS. Placental transmission of thyroidstimulating immunoglobulins. Br Med J 1975;ii:655-66.

4 Hollingsworth DR. Grave's disease. Clin Obstet Gynecol 1983;26:615-34.

5 Van Der Gaag RD, Drexhage HA, Dussault JH. Role of maternal immunoglobulins blocking TSH-induced thyroid growth in sporadic forms of congenital hypothyroidism. Lancet $1985 ; \mathrm{i}: 246-50$.

6 Holmes RC, Black MM, Dann J, James DCO, Bogal B. A comparative study of toxic erythema of pregnancy and herpes gestationis. Br J Dermatol 1982;106:499-510.

7 Holmes RC, Black MM. The fetal prognosis in pemphigoid gestationis (Herpes gestationis). Br J Dermatol 1984;110:67-72.

8 Barlow CF. Neonatal myasthenia gravis. Am J Dis Child 1981;135:209.

9 Plauché WC. Myasthenia gravis. Clin Obstet Gynecol 1983;26:592-604.

10 Perry CP, Hilliard GD, Gilstrap LC, Harris RE. Myasthenia gravis in pregnancy. Am $J$ Med Sci 1975;12:219-21.

11 Fraga A, Mintz G, Orozco J, Orozco JH. Sterility and fertility rates, fetal wastage and maternal morbidity in systemic lupus erythematosus. $J$ Rheumatol 1974;1:1293-8. 
12 Derve GS, Englert HJ, Harris EN, et al. Fetal loss in systemic lupus: association with anticardiolipin antibodies. $J$ Obstet Gynecol 1985;5:207-9.

${ }^{13}$ Lubbé WF, Butler WS, Palmer SJ, Liggins GC. Lupus anticoagulant in pregnancy. Br J Obstet Gynaecol 1984;91:357-63.

14 Hardy JD, Solomon S, Banwell GS, Beach R, Wright V, Howard FM. Congenital complete heart block in the newborn associated with maternal systemic lupus erythematosus and other connective tissue disease. Arch Dis Child 1979;54:7-13.

15 Scott JS, Maddison PJ, Taylor PV, Esscher E, Scott O, Skinner RP. Connective-tissue disease, antibodies to ribonucleo-protein and congenital heart block. $N$ Engl $J$ Med 1983;309:209-12.
${ }^{16}$ Litsey SE, Noonan JA, O'Connor WM, Cottrill CM Mitchell B. Maternal connective tissue disease and congenital heart block. Demonstration of immunoglobulin in cardiac tissue. N Engl J Med 1985;312:98-100.

${ }^{17}$ Mowbray JF, Gibbings C, Liddell H, Reginalt P, Underwood J, Beard RW. Controlled trial of treatment of recurrent spontaneous abortion by immunisation with paternatal cells. Lancet $1985 ; \mathbf{i}: 941-3$

M DE SWIET

Queen Charlotte's Maternity Hospital, and Brompton Hospital, London 